\title{
APUNTES SOBRE LA IDENTIDAD VIRTUAL DE GENERO
}

\author{
ANTONIO GARCÍA JIMÉNEZ \\ SONIA NÚÑEZ PUENTE \\ Universidad Rey Juan Carlos (Madrid)
}

Los medios construyen nuestras identidades influyendo en quiénes somos $y$ en los rasgos que nos definen grupalmente. Toda comunicación es, por tanto, identitaria. Los medios de comunicación difunden representaciones configurándose como un punto básico en la construcción de identidades, estableciendo finalmente un nosotros y un ellos, en conexión con los roles sociales y en dependencia del contexto político y económico, convertidos en objeto de pugna. Desde este punto de partida teórico, el propósito del artículo es considerar y analizar en profundidad las distintas relaciones que se establecen entre los conceptos de identidad, virtualidad y género en los medios de comunicación. La relación de la elaboración de diferentes modelos de identidad en los medios de comunicación con la tecnología, Internet y el feminismo es clara y ha sido objeto de numerosos estudios ${ }^{1}$. Esta relación se articulará como el eje teórico del artículo. Para mostrar esta relación en el contexto específico de los medios de comunicación se realizará un recorrido por los distintos presupuestos teóricos de la construcción identitaria ocupándonos, por tanto, de las identidades virtuales, de la conexión entre identidad y memoria y, por último, de la conexión entre identidad, género y virtualidad.

1. BACH, Marta; Altés, Elvira; Gallego, Juana (eds.): El sexo de la noticia, Barcelona, Icaria, 2002; BIJKER, Wiebe E. y LAW, John (eds): Shaping Technology / building Society: Studies in Sociotechnical Change, Cambridge, MIT Press, 1992; CARTER, Cynthia (ed): News, Gender and Power, Londres, Routledge, 1998; CoCKBURN, Cynthia: Gender and Technology in the Making, Londres, Sage, 1993; Cockburn, Cynthia: Machinery of Dominance. Women, Men and Technical Know-How, Londres, Pluto Press, 1985; Gallego, Juana: La prensa por dentro, Barcelona, Libros de la Frontera, 2002; LIE, Merete (ed): He, She and It Revisited. New Perspectives on Gender in the Information Society, Oslo, Gyldendal, 2003 y Zoonen, Van Liesbet: Feminist Media Studies, London, Sage, 1994. 


\section{IDENTIDAD Y MEDIOS DE COMUNICACIÓN}

Considerando el punto de vista mediático, Sampedro ${ }^{2}$ establece una primera clasificación de la identidad: hay identidades individuales, relacionadas con la trayectoria vital, y que son negociadas mediante procesos interpersonales y mediáticos, en combinación con estilos de vida; y también existen las identidades colectivas (en las que pesa fundamentalmente la pertenencia a un grupo). En este sentido Castells ${ }^{3}$ afirma que la sociedad de la información, o la sociedad red tal como la define el propio Castells, se encarga de satisfacer las necesidades de los individuos en relación a la identidad y el desarrollo personal. En esta sociedad red siguiendo a Castells ${ }^{4}$ es cada vez más habitual que las personas no organicen su significado en torno a lo que hacen, sino por lo que son o creen ser. Esta configuración de las identidades en la sociedad red permite que los agrupamientos, las comunidades virtuales, los individuos y organizaciones puedan interactuar de forma significativa en lo que Castells ${ }^{5}$ denomina la telaraña mundial de la comunicación interactiva. En este mundo multimedia se integrarían dos tipos de poblaciones muy distintas; los interactuantes y los interactuados, es decir, aquellos capaces de seleccionar sus circuitos de comunicación multidireccional y aquellos a los que se les proporciona un número limitado de opciones preempaquetadas. Y quién es quién vendrá determinado en buena medida por la clase, la raza, el país y, lo que es pertinente para nuestro análisis, el género tal como veremos en el desarrollo de la relación entre género y nuevas tecnologías.

En otro plano, nos encontraríamos con las identidades públicas (carácter que les confiere su visibilidad mediática), con diferentes jerarquías definidas a través de la cobertura y tratamiento mediático. Éstas se construyen a partir de la visibilización de las identidades personales y colectivas en grupos grandes de personas. Y se configuran, en gran medida, a través de los medios de comunicación, puesto que son quienes determinan a quién hay que representar y cuáles son las marcas que le definen. Así, hablamos de identidades mediáticas cuando se traducen, mediante los contenidos difundidos por los medios, en colectivas. De igual forma, la acción mediática consigue privatizar las identidades sociales.

Conviene no olvidar que gracias a los medios se normalizan o marginan unos atributos concretos dada su adecuación, o no, a las leyes o prácticas sociales. En este sentido, los medios priman las identidades oficiales, derivadas de aquellas instituciones sancionadas política y socialmente, de carácter educativo, legal, etc. Mientras, los medios también incluyen-crean identidades populares (que deben su nombre a la extracción de sus enunciadores y no tanto al nivel de aceptación general).

2. SAMPEDRO, Víctor: «Identidades mediáticas. La lógica del régimen de visibilidad contemporánea», Sphera Pública, 4 (2004), pp. 17-36.

3. CASTELLS, Manuel: The Information Age, Oxford, Blackwell, 1996.

4. Ibíd., p. 33.

5. Ibíd., p. 27. 
Las categorías de la jerarquía identitaria se completan con las identidades hegemónicas y las minoritarias, que se configuran en relación con el grado de acercamiento a las prácticas sociales más comunes. Dentro de las minoritarias (aquellas que escapan a lo oficial y hegemónico), Sampedro subraya la existencia de las identidades marginales (al margen de formas de representación impuestas por las instituciones o prácticas sociales) o incluso opositoras (consideradas así por enfrentarse a la adscripción identitaria asignada por las instituciones y la sociedad) ${ }^{6}$. En consonancia con estas palabras, las formas de construcción de esta jerarquización identitaria proceden de una lógica vinculada con los intereses de los medios de comunicación y tras la cual se encuentran diversas instancias de poder, los grupos de presión, así como la publicidad, en busca de las audiencias, o los propios informadores.

Tras esta exposición del contexto de jerarquía mediática, y sabiendo que la constitución mediática identitaria depende de la presencia o cobertura de los propios medios, es pertinente no olvidar que, de un lado, los medios imponen renovaciones continuas de las identidades, sin posible conexión con su pasado o bases de producción, en un claro proceso de descontextualización y con una falsa apariencia de neutralidad. Por otro, que la ubicación o estatus de cada identidad también depende de su valor en términos económicos y políticos. A veces, detrás del hecho de anteponer una identidad sólo se encuentra el afán de lucro escondido en mecanismos publicitarios encubiertos en otros discursos como el periodístico, y no solamente la búsqueda de grupos o audiencias concretas con identidades propias.

En lo que respecta a la televisión, Barker ${ }^{7}$ apunta la trascendencia de este medio, como un espacio propio en el que se llevan a cabo disputas culturales con dimensión global, lo que es especialmente importante por el impacto de las representaciones que difunde. Es palpable su papel en el germen de las identidades, dando lugar a la yuxtaposición de una amplia gama de discursos que incorporan, con fórmulas complejas, identidades culturales de carácter absoluto (étnicas o religiosas) o interculturales e híbridas.

Por otra parte, el repertorio de las identidades mediáticas también se construye en función del tipo de televisión. A esto han contribuido sus nuevos formatos, asociados habitualmente a niveles medios y altos económicos, lo que permite un consumo mayor en medios que transmiten una gama más amplia de representaciones identitarias. Asociada a la televisión global-local, y favorecida por la misma, se ubica un doble proceso con impacto tanto regional como transnacional que tienen su huella en el surgimiento o potenciación de nuevas identidades.

En último extremo, la apuesta de Barker es clara: partiendo de Rorty ${ }^{8}$, aboga por el diálogo como método, y por que la televisión se convierta en «una esfera

6. SAMPEDRO, Víctor: Op.cit.

7. BARKER, Chris: Televisión, globalización e identidades culturales, Barcelona, Paidós, 2003.

8. RORTY, Richard: Objetividad, relativismo y verdad: escritos filosóficos, Barcelona, Paidós, 1996. 
pública de ciudadanos diversa y plural, en la que la ciudadanía como forma de identidad se convierta en terreno abonado para una política compartida. Es decir, la mejor oportunidad para desarrollar un proyecto identitario privado puede ser vivir en una cultura que se precie de ser heterogénea»". Una identidad ciudadana que estaría directamente vinculada a la democracia, de modo que se permitiera unir las identidades culturales (nivel micropolítico) y los derechos políticos (nivel macropolítico).

Hasta aquí el apartado las representaciones. En el plano de lo que ve la audiencia o el público, entendemos que la identidad se difunde pero no se transmite de forma directa, puesto que siempre se produce una negociación entre las audiencias y los contenidos emitidos, en la que intervienen matrices culturales, cognitivas, económicas (que influyen en la determinación de los significados que las personas atribuyen a lo que ven, leen y oyen en los medios), dando lugar a diversas de asimilación e interpretación. De resultas de este doble proceso de representación de las clases sociales, la nación o la edad (en contextos tanto de ficción y de no ficción), y de deconstrucción y cuestionamiento multivariable respecto de los significados mediáticos por parte de las audiencias, se conforman las identidades.

En lo que se refiere a la relación entre identidad de género y comunicación, entre la prolija literatura sobre el particular, no deberíamos olvidar, en términos generales, la infrarrepresentación de la mujer en los medios, así como su ubicación en roles estereotipados que repiten las formas tradicionales de imposición patriarcal, demostrada en diversas formas, tales como el tratamiento de la violencia doméstica, que se revela como un caso prototípico de salto de la esfera privada a la pública en los últimos años. El propio Sampedro ${ }^{10}$ argumenta del siguiente modo la construcción de la identidad femenina:

"la identidad femenina hegemónica aún sigue definida, en gran parte, por su capacidad de despertar el deseo del varón. Las escasas películas que tienen a una mujer lesbiana como protagonista se destinan a mercados muy reducidos (el «cine de gays y lesbianas») o poco legitimados (la pornografía). Se entiende, por tanto, que una lesbiana no aportará elementos atractivos ni de identificación a quienes no sientan complicidad emotiva o excitación sexual».

\section{IDENTIDADES VIRTUALES}

Estamos convencidos de que en las próximas décadas lo digital se habrá integrado tanto en los imaginarios colectivos como en los individuales en sus prácticas de sentir o manifestar la identidad. Tomando como punto de partida que la tecnología es básica para entender la identidad tanto en el plano personal como colectivo, nos aproximamos a la conexión entre Internet y la identidad de dos formas. Una primera que recoge la relación entre identidad, memoria e

9. BARKER, Chris: Op. cit., p. 250.

10. SAMPEDRO, Víctor: Op. cit. 
Internet. Y una segunda que se aproxima sucintamente a la influencia en estos procesos de esta tecnología.

\subsection{Identidad y memoria}

Es evidente la relación de la identidad con la memoria y con las formas de la rememoración. En especial, si tenemos presente la influencia cultural de la tecnología a la hora de reconocernos y de fijar nuestras pertenencias simbólicas. De hecho, "la memoria (...) debe entenderse más bien como un conjunto de prácticas, a través de las cuales los sujetos van construyendo la propia identidad o, tal vez mejor, van elaborando la propia biografía» ${ }^{11}$.

Por su parte, García Gutiérrez ${ }^{12}$ entiende que la identidad personal se define como un proceso de carácter dinámico que se deriva de interacciones difusas a partir de la memoria, junto a la cultura, la educación, el conocimiento, el entorno, la imaginación e incluso lo físico. La memoria se presenta, pues, como un factor clave en la esfera de las identidades colectivas, como es la nacional. Para este autor, la presencia de lo nacional es radical tanto en la configuración de la identidad o identificación como en los procesos de creación de las diferentes memorias. En efecto, los sentidos o significados que proponen se basan en la mitificación del pasado con la intención de convertirlo en natural.

En gran medida, en una época dominada por una evidente aceleración del tiempo y compresión del pasado (con su correspondiente grado de frustración), «la memoria, al igual que ese otro fenómeno, tan característico de nuestro tiempo, que es la musealización generalizada, son invocadas para que se constituyan en baluartes que nos protejan del miedo vertiginoso a que las cosas devengan obsoletas y desaparezcan ${ }^{13}$, de tal suerte que, gracias a la memoria, el ser humano podría afrontarlo revertiendo estos procesos. En realidad, de acuerdo con la crítica de Virilo ${ }^{14}$, para que la memoria pueda trabajar en pos de la identidad colectiva requiere tecnologías rápidas que, de modo paradójico, den lugar a procesos culturales con el ritmo y tiempo adecuado.

Y a la memoria le sigue el olvido. Una memoria, y el consiguiente olvido, de un pasado que no existe. $O$ dicho de otra forma, un pasado que no es cognoscible, o lo es con grandes limitaciones, habida cuenta de que necesita de la interpretación mediante un discurso que está anclado en el tiempo presente y que presenta un gran afán de autorepresentación. Lo que, por otra parte, nos debiera exigir el esfuerzo de rememorar por nuestra cuenta, puesto que, de lo contrario, serán otros quienes nos recuerden lo que debe ser conservado.

11. CRUZ, Manuel: Las malas pasadas del pasado. Identidad, responsabilidad, historia, Barcelona, Anagrama, 2005, pp. 154-155.

12. García GutiérReZ, Antonio: Fijaciones. Estudios críticos sobre políticas, culturas y tecnologías de la memoria, Madrid, Biblioteca Nueva, 2005.

13. CruZ, Manuel: Op. cit., p. 125.

14. VIRILO, Paul: El cibermundo. La política de lo peor, Madrid, Cátedra, 1999. 
Curiosamente, a juicio de Vattimo ${ }^{15}$, los órganos que más influyen en estos procesos discursivos que aluden al pasado son los medios de comunicación. Se convierten en auténticos órganos de la historización que inducen a la pérdida de la memoria mediante la continua reivindicación sobre el pasado, de tal suerte los sujetos pensaríamos el pasado de forma diferente dado que se re-presenta en sucesivas y múltiples ocasiones.

En este sentido, Internet se postula como un conglomerado tecnológico que es y dará cobijo a la memoria de la humanidad y que muestra una clara habilidad para homogeneizar, reducir y acelerar. Se postula como un espacio necesitado de una visión crítica que ponga de manifiesto las formas de monopolio y colonización cultural y de conocimiento que se difuminan en su interior. Desde donde se podrá valorar adecuadamente las interrelaciones entre identidad, conocimiento y memoria. Si bien, «ni el poder, ni la tecnología, ni la cultura dominantes se reservan el secreto de la identidad o el control de la elección de pertenencias simbólicas, mucho menos desde la imposición de cuadros conductuales o inventarios de tópicos culturalistas que sólo sirven para tertulias académicas» ${ }^{16}$.

Como ya se ha visto, la identidad está conectada con la memoria (que a su vez también es una práctica cultural) y con la rememoración, escapando, en cualquier caso, de modos prefijados y con rasgos únicos. La memoria permite crear redes híbridas de sentido, de la mano de la invasión digital que presupone nuevos modos de comprender el mundo presente y pasado.

De tal suerte que convivirán diversas memorias: siempre existirán memorias oficiales y memorias de la clandestinidad. Y devendrán en exomemorias (también interiorizadas) al amparo de lo digital, activándose los correspondientes procesos de imaginación y recuerdo. García Gutiérrez ${ }^{17}$ propone una clasificación de memorias digitales sociales, basadas, unas, en la ordenación y evocación del pasado común y, otras, en el individual o compartido:

1. Las personales. Las que se pueden encontrar en cajones, paredes o álbumes y devienen en repertorio ordenado de objetos para la «evocación». Mientras, las grupales trascienden el control de los individuos, anclándose en lo emotivo: en la familia, el grupo de amigos, o el barrio.

2. Las comunitarias-territoriales con una fuerte base geográfica o geosimbólica, donde el lugar es el centro de intereses compartidos: de afectos, tradiciones, valores o puramente económicos.

3. Las comunitarias en torno a pertenencias como las asociaciones a favor de una u otra cuestión social, de lucha contra enfermedades, etc. Aquí es el objetivo (abierto y dinámico) lo que se comparte, provocando la proliferación de las identificaciones, lo que no descarta posibles contradicciones entre las vinculaciones, por ejemplo, personales. De igual forma, con el tiempo

15. VATtimo, Gianni: La sociedad transparente, Barcelona, Paidós, 1990.

16. García Gutiérrez, Antonio: Op. cit., p. 14.

17. Ibíd., p. 43. 
adquirirá mayor relevancia el valor de lo virtual, con todas las opciones de suplantación que se abren.

4. Las sociales, entre las que cabría incluir las locales, nacionales, estatales o mundiales (de carácter político, religioso, etc.) en propagación mediante instrumentos como la ecuación.

\subsection{Nuevas identidades}

En primer lugar, acudimos al trabajo de Helen Kennedy ${ }^{18}$, que hace un mapa de la investigación sobre la identidad en Internet, situándolo dentro de los estudios de los nuevos medios, y en medio de la crítica a la idea de Turkle ${ }^{19}$ de que una de la claves de las identidades virtuales es su anonimato. Para Kennedy, las identidades en línea son, a menudo, continuación de las mismas offline, y no tanto versiones reconfiguradas de las subjetividades en la vida real. De ahí que haya que ir más allá de las identidades en Internet y echar una mirada a los contextos offline.

El estudio clásico de Turkle ha influido en la forma de plantear la relación entre la construcción de la identidad y las tecnologías en red. Básicamente, las identidades en el ámbito online serían fluidas, fragmentadas y harían posible nuevas formas de identidad. Si bien es una obra con diferentes lecturas, ha prevalecido esta posición en la literatura sobre el particular. Frente a esta propuesta teórica, Kennedy aporta estudios empíricos etnográficos y biográficos que concluyen que las personas en Internet no gozan de una identidad virtual fragmentada sino estable. Es decir, estaríamos hablando de ciberidentidades unificadas.

En su crítica el concepto de anonimato, recurrente en este ámbito, destaca su acusación de lo limitada que es la exploración de las diferencias significativas entre las condiciones de ser y sentir (no es lo mismo ser que sentirse anónimo, además de presentarse en diferentes grados). Desde esta posición, la idea que subyace en el trabajo de Turkle es que una de las claves más importantes se encuentra en el anonimato, ya que si no se conoce al otro, se produce automáticamente un proceso de igualación. Sin embargo, en palabras de Kennedy, hay trabajos empíricos que restan importancia a esta cuestión. También pone bajo sospecha otras aproximaciones a este tema, por ejemplo, en lo que atañe a algún de clasificar las identidades en función de su dominio virtual (homepages, foros), tal y como propuso Tetzlaff ${ }^{20}$.

En términos generales, entiende que, en el campo de Internet, no se ha asimilado parte del rico debate que se ha desarrollado en los estudios culturales so-

18. KENNEDY, Helen: «Beyond anonymity, or future directions for internet identity research", New media \& Society, 8:6 (2006), pp. 859-876.

19. TURKLE, Sherry: La vida en la pantalla. La construcción de la identidad en la era de Internet, Barcelona, Paidós, 1997.

20. Tetzlaff, David: «Y-Ho-Ho and a Server of Warez: Internet Software Piracy and the new global information economy", en A. Herman and T. Swiss (eds.): The World Wide Web and Contemporary Cultural Theory, London, Routledge, 2000, pp. 99-126. 
bre el problema de la identidad de mano de autores como Braidotti. Finalmente, plantea la necesidad de afrontar una profunda reflexión sobre las implicaciones de cuestiones como la posibilidad de fingimiento, la identificación o la práctica nómada, sin dejar de lado el grado de utilidad del concepto de identidad para la investigación de los nuevos medios.

Como se puede observar, son múltiples las derivaciones prácticas y teóricas que se pueden extraer de este asunto. A título ilustrativo y en el marco de las comunidades virtuales, Aguilera ${ }^{21}$ analiza, en el seno de una comunidad virtual de chilenos, los vínculos sociales al objeto de establecer los procesos de construcción de esta forma de identidad colectiva virtual. A priori, se ubica en la posibilidad de que, en los espacios textuales de interacción como los chats y los foros, muten las vinculaciones entre los miembros de la comunidad. Apostando por los proyectos e intereses compartidos en detrimento de las relaciones familiares y territoriales. Este estudio se centra en lo que se puede denominar espacios textuales de interacción como los chats y los foros para ver las concomitancias entre la comunidad virtual que se construye en la práctica y la comunidad virtual ideal.

Sus conclusiones apuntan a una comunidad virtual en la que el criterio más importante de pertenencia es la identificación nacional chilena. Según los criterios de pertenencia, se hace referencia a la identidad nacional implicándose en esta comunidad virtual tanto las relaciones online como las relaciones offline. Gracias a este estudio se observa que el nivel de interacción es muy bajo. De los 90.000 miembros potenciales, sólo una treintena de socios realmente interacciona. La gran mayoría se limita a leerlo.

Por otra parte, en los foros, a pesar de lo que se podría imaginar en un primer momento, al enlazar las diversas experiencias, hay conflictos, jerarquías y tensiones. Recordemos que las dinámicas de poder se entrecruzan en cualquier caso. En los foros, la estructuración de los mensajes es poco ordenada. Esto puede estar unido a la existencia de diferentes marcos de comprensión y las diversas situaciones y precedentes de cada uno de los mensajes que se encuentran en la comunidad. Por último, en los chats, como espacio de mediación en tiempo real (con unas relaciones diferenciadas caracterizadas por el anonimato lo que puede derivar en una nueva forma de sociabilidad), son constantes las referencias a la vida real. Es patente el papel fundamental que se adscribe a la comunidad preexistente y a los núcleos familiares, de suerte que la comunidad virtual se constituiría como una continuación de la vida fuera de la pantalla. Es decir, una comunidad virtual idealizada que, en esta ocasión, refuerza lo nacional.

Hay otros lugares de reunión en Internet, alrededor de otros ámbitos de interés. Así, se puede encontrar, en el nivel de los fans, comunidades basadas en web con chats, grupos de discusión, foros, etc., con múltiples tipos discursivos,

21. Aguilera Valpuesta, Francisco: «Sociabilidad virtual, vínculo social y construcción de la identidad. El Caso de la comunidad virtual El Rincón Chileno", II Congreso online del OCS, 2004. En www.cibersociedad.com/congres2004 
tales como evaluaciones, exégesis, críticas, análisis históricos, etc., hecho que cuenta con un alto y rápido crecimiento.

Detengámonos en un caso. A cuentas de Futurama ${ }^{22}$, que es una serie de dibujos animados difundida en general por televisión, se ha creado una interacción virtual y amorfa, cuya interconexión depende de los ritmos de la programación de las televisiones, que no están fijadas a un tiempo específico. Además de diferencias en lo que atañe a las dinámicas temporales, con dispares percepciones, se puede percibir la amplia gama de formas de participación, y que las discusiones tienen lugar tomando en consideración el espacio material, la esfera doméstica y el espacio virtual de la cibercomunidad.

El cibermundo de Futurama es otra demostración de la interacción entre televisión e Internet. A su vez, resulta paradójico puesto que combina espacios que están considerados altamente convencionales con espacios asociales, radicalmente abiertos y libres respecto de la mayor parte de la regulación social. En cierta medida, pone de manifiesto la creciente fluidez del sujeto y su carácter híbrido, la simultánea oportunidad de nuevas de auto-construcción y autorealización, que se proponen como amenaza a la estabilidad subjetiva.

Precisamente, Internet puede ayudar a conocer el juego identitario en sus diversas modalidades. Así, en el interesante trabajo de Campbell123 sobre los grupos de discusión de skinheads, se detecta que el racismo no es visto como un componente de la identidad de este tipo de grupo, sino que el compromiso se extiende al concepto de identidad blanca. Las narrativas sobre lo blanco que se articulan a través de la Red, revelan la relevancia de lo racial y corporal en el mundo virtual. En este estudio, los skinheads no representan un racismo discriminatorio explícito. Más bien imaginan lo blanco como una condición preformativa de su propia comunidad, una noción que necesita una figurada, y real, relación agresiva con la otredad, con los otros. Un otro que no es masculino o macho, duro y heterosexual.

Internet es un como campo de pruebas de la identidad, también para la época adolescente. En el estudio de Valkenburg, Schouten y Peter ${ }^{24}$ se corroboran $^{2}$ los postulados de la teoría de la identidad de los adolescentes: para los más jóvenes es un tiempo dedicado a la consideración de uno mismo y de su identidad, con su correspondiente experimentación. Los adolescentes de menor edad usan Internet con más frecuencia para comunicarse con extraños y jugar con su propia identidad. Por su parte, los teenagers de mayor edad acuden a Internet más a menudo con vistas a comunicarse con su red de contactos personales. $Y$ aunque las estrategias no difieren en exceso, los de menor de edad simulan tener más edad.

22. BAILEY, Steven: Media audiences and identity. Self-construction in the fan experience, New York, Palgrave MacMillan, 2005.

23. Campbell, Alex: "The Search for authenticity», New Media \& Society, 8:2 (2006), pp. 269-294.

24. Valkenburg, Patti M.; SChouten, Alexander P. and Peter, Jochen: «Adolescents' identity experiments on the internet", New Media \& Society, 7:3 (2005), pp. 383-402. 
Desde el punto de vista de género, también se encontraron diferencias. En comparación, las chicas experimentaron con su identidad más a menudo al objeto de explorarse a sí mismas e investigar cómo aparentan ser a los ojos de los demás. Este hecho tiene que ver con la relación negativa que presentan con su propio cuerpo, lo que es un tema de preocupación más importante. Después de la edad y del género también incumbe el grado de introversión de la persona. De hecho, las chicas y los adolescentes introvertidos experimentan con su identidad, por razón de compensación social, más a menudo que los chicos y los adolescentes extrovertidos.

Para finalizar, destaquemos que cada vez es más fácil cultivar una identidad flexible en el marco del ciberespacio, aunque con la sombra de renovadas prácticas de poder, lo que, como ya comentamos, afecta a la sociedad y a la memoria individual y colectiva. Y una de las cuestiones más importantes se sitúa en la compleja relación entre las identidades online y las identidades offline. Más fluidas las primeras, pero siempre presentes, tanto en el plano virtual como en el no virtual, si bien son limitadas, reorganizadas y en ocasiones negadas por las dinámicas sociales en sus diferentes formatos.

\subsection{Identidad, género y virtualidad}

Según Jiménez Cortés ${ }^{25}$, la identidad de género es semejante al resto de identidades en la medida en que es el producto de la dialéctica entre los sujetos y los mundos culturales. Su construcción depende de un doble pilar: por un lado, la opresión en los ámbitos social, político o económico sobre la mujer, frente a la posición dominante del hombre en un mundo que puede ser interpretado a partir de "pares opuestos». Por otro, la identidad de género también tiene que ver con la idea de pertenencia a un mismo grupo, a través de vínculos de tipo personal.

La identidad de género se construye, participando, al mismo tiempo, en la interpretación del mundo y en sus correspondientes significaciones. En gran medida, la masculinidad y la feminidad son entendidas como modelos sociales excluyentes, que están unidos tanto a las identidades personales como a la configuración, en el marco de las interacciones sociales, de las identidades colectivas.

Excede a este trabajo ofrecer el panorama completo de la ingente cantidad de producción científica, tanto nacional como internacional, que desde la comunicación estudia el género o viceversa. No obstante, a modo de resumen imposible, acudimos al trabajo de Larrondo ${ }^{26}$ que entiende que, desde una perspectiva feminista, el ámbito de la comunicación se presenta como una

25. JiMÉNEZ CORTÉS, Rocío: «Internet y educación: La gestión de la identidad cultural de género», $1^{\circ}$ Congreso Online del Observatorio para la Cibersociedad, (2002). En http://www.cibersociedad.net/ congreso/comms/g15jimenez.pdf.

26. LARRONDO URETA, Ainara: «La Red al servicio de las mujeres. Aproximación a la relación mujer y medios de comunicación en Internet», Estudios sobre el Mensaje Periodístico, 11 (2005), pp. 375392. 
pieza clave para: en primer lugar, influir en la agenda y en el posicionamiento de la mujer en las noticias y, en segundo lugar, para sensibilizar a la mujeres al respecto de esta realidad, sin excluir a los hombres.

Desde esta misma mirada, Internet se presenta "como herramienta cultural que media entre le sujeto y los patrones sociales entre los que se encuentran los referentes al orden social genérico. Internet actúa como instrumento cultural que institucionaliza valores, códigos de acción y discursos $\aleph^{27}$.

En consecuencia, el ciberespacio se desplegaría como un nuevo espacio público en el que se sitúa la necesidad de adquirir un mayor grado de visibilidad y de potenciar la aparición de medios alternativos a los tradicionales a través de los cuales se pueda canalizar la defensa del reconocimiento de la mujer. En efecto, Internet se presenta como una opción que potencia la posibilidad de nuevos modelos de difusión ${ }^{28}$ :

"Internet pone al alcance del movimiento feminista potencias expresivas desconocidas, entre otras, una nueva significación de comunicación colectiva que convierte a las mujeres en autoras, transmisoras y destinatarias de información. A su vez, el abaratamiento e internacionalización de la comunicación en red facilita que grupos de mujeres de todo el mundo se interrelacionen para formular estrategias de cohesión o, simplemente, intercambiar experiencias y apoyos en los cada vez más numerosos espacios comunicativos virtuales».

En este sentido, los distintos trabajos feministas sobre género y tecnología han oscilado en las últimas décadas entre posiciones optimistas y pesimistas al respecto de los de los beneficios de la tecnología y, específicamente, por lo que respecta a las implicaciones políticas y activistas de este debate académico. El trabajo de Valerie Solanas en la década de los 60 y los 70 se muestra muy optimista acerca de la potencialidad liberadora de la tecnología. De hecho el SCUM Manifesto ${ }^{29}$ (1968) de Solanas advierte de que las nuevas tecnologías son clave para liberar a la mujer de su cuerpo biológico y de la dependencia económica del hombre. En 1970 Shulamith Firestone publica The Dialect of Sex. The Case for Feminist Revolution que se convirtió en un texto esencial para las feministas radicales. Desde su doble posición como teórica y activista, Firestone contribuyó de manera relevante a las nuevas aproximaciones de la teoría feminista y de la Sociedad de la Información. Firestone clamaba por la liberación de la mujer de la tiranía de la biología. Su trabajo se vio influido no sólo por el de feministas como Beauvoir, sino también de teóricos de la Sociedad de la Información como Daniel Bell que compartían con Firestone su optimismo acerca del papel que el automatismo desempeñaría en la erradicación del trabajo mecánico y de la división de género. La tecnología crearía así las condiciones necesarias para desterrar las condiciones naturales opresivas de las mujeres en la sociedad.

27. JiMÉNEZ CORTÉS, Rocío: Op. cit.

28. LARRONDO URETA, Ainara: Op. cit., p. 38

29. SolanAS, Valerie: The SCUM Manifesto, Londres, Verso, 2004. 
Ya en la década de los 80 algunas feministas como Cynthia Cockburn ${ }^{30}$ inician una corriente teórica más pesimista en cuanto a la potencialidad liberadora de la tecnología para las mujeres. En su trabajo, basado en el análisis empírico de los lugares de trabajo, Cockburn argumenta que la dominación masculina de las habilidades técnicas y del conocimiento tecnológico sirve para mantener una división sexual del trabajo. Cockburn aboga, de este modo, por un movimiento autónomo de mujeres para transformar la naturaleza de la tecnología. Se planteaba, entonces, la cuestión de si las mujeres experimentan la tecnología como opresiva porque los hombres dominaban el uso de la tecnología o si la tecnología era inherentemente patriarcal. La respuesta a esta cuestión por parte de las feministas liberales es que los hombres dominaban el uso y diseño de la tecnología y, por tanto, habría que implicar más a las mujeres en el desarrollo de la tecnología. Por su parte, las feministas radicales situaban la raíz del problema en la naturaleza patriarcal de la tecnología y promovían, por tanto, el rechazo total de la tecnología. El Manifiesto for Cyborgs de Dona Haraway ${ }^{31}$ introduce una mediación en este debate polarizado $y$, reaccionado en parte contra el feminismo radical, Haraway afirma que la tecnología es una parte esencial de nuestra identidad. Su argumento es que la tecnología es central para nuestra vida diaria y que, por tanto, puede resultar útil concebirnos a nosotros mismos como cyborgs y usar esta conceptualización como un instrumento para cambiar las relaciones de poder e identidades en relación a la tecnología. El trabajo de Haraway abrió nuevas posibilidades para el análisis feminista y político para internarse en un proceso más positivo explorando el modo en el que las vidas de las mujeres están íntimamente conectadas con las tecnologías, aún con todas las contradicciones y complejas relaciones de poder que se establecen en este proceso. En este sentido el trabajo de Manuel Castells ${ }^{32}$ obvia, en cierto modo, las cuestiones de desigualdad para centrarse en la creencia de que la tecnología tiene un poder transformador de la sociedad. En este aspecto el trabajo de teóricos sociales como Castells se relaciona con el de feministas como Sadie Plant ${ }^{33}$ que se mueven más allá de los aspectos negativos de la tecnología y enfatizan la existencia de espacios libres para que las mujeres desarrollen nuevas identidades mediante la tecnología. Aparece, de este modo, en el trabajo de Plant la idea de las identidades fluidas que desafían aquellas identidades que estaban prefijadas por el patriarcado. En este sentido, teóricas como Sherry Turkle apuntan las posibilidades que tiene la tecnología para expresar múltiples y, a menudo inexplorados, aspectos de la identidad, para poder establecer así una suerte de juego con la identidad e intentar, de este modo, crear nuevas identidades. Precisamente, en la visión de la tecnología como un elemento clave para el desarrollo de nuevas identidades, que pueden ser de resistencia, se encuentra

30. Cockburn, Cynthia: Machinery of Dominance. Women, Men and Technical Know-How, Londres, Pluto Press, 1985.

31. HaRAWAY, Donna: «Manifesto for cyborgs», The Socialist Review, 7 (1985), pp. 115-135.

32. CASTELLS, Manuel: Op. cit., p. 1.

33. Plant, Sadie: Zeros + Ones, Londres, Fourth Estate, 1997. 
la intersección entre la teoría feminista y la teoría social. Por ello, y tal como hemos venido argumentando en nuestro artículo, la tecnología juega un papel fundamental en la construcción y determinación del género, la identidad y otras relaciones sociales ${ }^{34}$.

\subsection{Género, identidad y ciberfeminismo}

La clave para entender la identidad de género en Internet se sitúa en lo que se denomina ciberfeminismo. Sanz ${ }^{35}$ lo ubica en el seno de una doble interrelación: los estudios de Ciencia, Tecnología y Sociedad y los estudios feministas sobre tecnologías. Lo que se ha traducido en la incorporación de una visión constructivista en el análisis de las tecnologías por parte de las feministas. Y, también, en la inclusión del género como categoría y variable de estudio en la reflexión sobre la sociedad y la tecnología. Curiosamente, de esta aportación se podría deducir una actitud sexista al tener que insistir, desde posiciones feministas, no sólo en la necesidad sino también en la pertinencia epistemológica de su consideración desde un punto de vista analítico.

Hagamos historia. Dentro del campo del feminismo cultural, aparece un sector preocupado por las tecnologías de la información que es el ciberfeminismo (término que ve la luz en 1991 gracias el grupo australiano VNS Matriz, que se preocupa por la identidad y sexualidad en el ciberespacio). En términos generales, su análisis va desde la atención sobre el corte patriarcal de las tecnologías e Internet hasta sus potencialidades en tanto que espacio abierto, difuso y no jerárquico.

Con anterioridad, se puede señalar una primera etapa a finales de los $60 \mathrm{y}$ principios de los 70 caracterizada por su marcado carácter optimista al hacer hincapié en el potencial liberatorio de la tecnología. Por su parte, durante los 80 , la discusión se planteó alrededor de la causa de que la experiencia tecnológica de la mujer sea opresiva. La pregunta es si la dominación a través de la tecnología se deriva del hecho de que los hombres dominan su uso o del hecho de que la tecnología es inheremente patriarcal.

Esta disyuntiva fue puesta en entredicho en el pensamiento de Haraway (en su "Manifesto for Cyborgs») al rechazar una posición y otra. Concretamente, se muestra optimista en cuanto a las oportunidades de transformación política y, de igual modo, subraya una idea clave: la tecnología es parte de nuestras identidades, lo que coincide con postulados exógenos a los estudios de género. Desde este punto de vista, se abren nuevas posibilidades para el análisis feminista y para la acción política, tras la necesaria exploración de las modalidades de conexión entre las mujeres y las tecnologías, pese a su carácter diverso, complejo $\mathrm{y}$, a veces, contradictorio.

34. WYATT, Sally: «Feminism, technology and the information society. Learning from the past, imagining the future", Information, Communication \&Society, 11:1 (2008), pp. 111-130.

35. SANZ GONZÁleZ, Verónica: «Las tecnologías de la información desde el punto de vista de género: posturas y propuestas desde el feminismo», Isegoría, 34 (2006), pp. 193-208. 
Ya en los 90, Sadie Plant emerge con una carga positiva, destacando no sólo las oportunidades que las tecnologías ofrecen a la mujer, sino poniendo de manifiesto las borrosas fronteras entre lo masculino y lo femenino, entre hombre y máquina. O dicho de otra forma, minimiza las contradicciones de la Sociedad de la Información, para enfatizar los espacios disponibles en la esfera de la mujer. En este punto, entiende que el ciberespacio se encuentra más cercano a la identidad feminidad puesto que goza de valores cercanos a la mujer. Mientras, Turkle, tal y como ya hemos apuntado en un apartado anterior, destaca la capacidad de experimentación y creación de los variados aspectos del sujeto y la subjetividad que dan lugar a las identidades virtuales.

Frente a estas opiniones, se alzan voces discordantes (como la de Wacjman ${ }^{36}$ ) que critican esta visión utópica de un espacio en el que es posible reconfigurar las viejas identidades. En el artículo de $\mathrm{Sanz}^{37}$, se agrupa estas críticas procedentes de otros sectores del ciberfeminismo:

1. Frente a las comunidades virtuales, las comunidades e identidades off line siguen pesando claramente. De hecho, son evidentes los múltiples casos y formas de brecha digital en función del país, clase social, y género.

2. Las situaciones de desigualdad se mantienen y en muchos casos se agrandan. Los valores e identidades que hipotéticamente se debieran eliminar se desplazan a la Red, con un efecto rebote que hace que aumente el impacto de los mismos.

3. Tras formas culturales en principio liberadoras, como la cultura hacker, (mitificada por determinados sectores como una forma de emancipación), se esconden realmente formas culturales tradicionales. En el caso de la identidad hacker, estaríamos ante un sustrato cultural de base «masculina, blanca y de clase media».

4. No se ha analizado convenientemente la falta de presencia de mujeres en el diseño ni en la producción de contenidos, ni sus consecuencias, en el ciberespacio.

Tras este repaso cronológico del ciberfeminismo, y a modo de resumen, este término en la actualidad aparece con tres usos ${ }^{38}$ : en primer lugar, en relación a la utilización del ciberespacio y de la tecnología en red para desarrollar los postulados feministas y poder conseguir sus objetivos; en segundo lugar, el estudio, por parte las feministas, de la presencia del modelo patriarcal en las propuestas tecnológicas y en sus diversos usos; en tercer lugar, la posibilidad de crear comunidades en las que las mujeres puedan re-construir la propia identidad, tras superar las bases de los modelos de dominación. Desde este prisma, todavía hay muchas batallas que librar. Alguna de ellas, aunque de carácter teórico, resultan relevantes en el plano identitario y están relacionadas con la pertinencia de incluir la teoría feminista en: la reflexión sobre la privacidad y

36. WajCman, Judy: Technofeminism, Cambridge, Polity Press, 2004.

37. SANZ GONZÁLEZ, Verónica: Op. cit.

38. LARRONDO URETA, Ainara: Op. cit. 
en el desvelamiento de supuestas identidades liberadoras (como la hacker) en el imaginario popular de alto nivel tecnológico detrás de la que subyace una clara identificación con lo masculino y lo occidental.

Sin entrar a detallarlas, existe un gran número de iniciativas virtuales y redes ciberfeministas que han ocupado Internet, y que se ocupan de luchar por conseguir la igualdad, a través de: la participación en ámbitos de comunicación como son los foros o los chats; de servicios de información, con noticias y todo tipo de documentos, que pretende cubrir las opciones informativas de las mujeres en Internet desde todos los puntos de vista; y de diferentes formas de servicios, que les sirve de apoyo. Su objetivo es hacer más libre y participativo el ciberespacio, en el que el componente de género forme parte vital y en el que tenga cabida la formación, la reflexión y el diálogo alrededor de la mujer. En este sentido, resulta de especial relevancia el trabajo de Margaret Andrews ${ }^{39}$ que explora las prácticas ciberfeministas, en España destacando el carácter político relacionado con la creación de nuevas identidades que se manifiesta en la praxis ciberfeministas de feministas como Boix o Bertomeu.

Para finalizar, en lo que afecta al eje de este escrito, acudimos a Remedios Zafra ${ }^{40}$ quien, con una mirada perspicaz alude a la conexión entre virtualidad, cuerpo e identidad. Desde su punto de vista, podría parecer paradójico que en las sociedades actuales, cuando lo virtual tiene una mayor presencia, todo lo que esté relacionado con el cuerpo, con lo físico, está radicalmente presente en la configuración de la propia identidad. Y esto ocurre porque ambas facetas se definen mutuamente, son una misma realidad. De ahí que no sea de extrañar la exaltación de lo corporal de forma simultánea a la diseminación del mundo virtual. En este contexto, Internet aparece como una auténtica industria de las identidades y un espacio de la subjetivación, de tipo post-corpóreo, en el que se difuminan los límites de lo real y de lo que no es, de la simulación y de la realidad. Es la pantalla la que da la apariencia y no la piel.

No sólo estas múltiples identidades creadas en Internet estarán conectadas con la escisión corporal, sino que también mantienen un alto grado de vinculación con la suspensión de la autoridad. Y es a partir de ahí cuando se genera parte de la sublimación de las formas de prohibición y represión. Siempre frente al poder desplegado al hilo de la identidad del sujeto que actúa y que concurre en la identidad del otro.

Las identidades en Internet pueden modularse en diversas identidades textuales. En primer lugar, nos encontramos con las identidades coincidentes, que se adhieren a las personas que se comportan y hablan del mismo modo dentro y fuera del ciberespacio, aunque con la correspondiente aceleración propia de Internet, de lo que se deriva una mayor intensidad. A continuación, las identidades imaginarias se articulan cuando el sujeto se inventa una persona que, en

39. ANDREWS, Margaret: "Ethics, Gender and the Internet: An exploration of some Spanish feminists' praxis", Journal of Spanish Cultural Studies, 7: 1 (2006), pp. 37-49.

40. ZAFRA, Remedios: "Apuntes sobre feminismo y subjetividad en Internet", Mujer y cultura visual. En http://2-red.net/mcv/pensamiento/tx/text_rz6.html 
principio, no tendría nada que ver el propio sujeto. Se trata de una identidad que requiere de un especial denuedo, por lo que suele estar limitado en el tiempo, salvo que se ayude de simulaciones del tipo de Second Life o lo SIMs. Finalmente, las identidades simbólicas son auténticas transformaciones en forma de máscara que permite proyectar a cada persona una imagen de sí, en muchos casos más cercana, real y vinculante que la que se da fuera de la Red, como si de un acto liberador se tratara.

Sin embargo, este sistema identitario que se da en ciberespacio es y "seguirá siendo heredero de una asimetría fundacional (hombre y mujer como sujeto y objeto, agente e instrumento) que se ratifica mediante la obstinación de los mitos patriarcales en el terreno de los intercambios simbólicos (relaciones de producción y reproducción) generadas también en Internet» ${ }^{41}$.

La escapatoria se encuentra a medio camino entre la identidad imaginaria y la simbólica, trocadas en piezas clave para hacer y deshacer el género. Pese a que el referente patriarcal intentará estar presente, este proceso es posible encontrándose con el pasado y con el ser. El problema es claro. La gran cantidad de roles exige, muchas veces, un ansia de estabilidad sobre la que siempre girará el fantasma de lo patriarcal. Así, el reto y la reafirmación feminista sobre la identidad y la subjetividad virtual queda emplazado a una doble labor: desplazar la imposición identitaria patriarcal, evitándola, y «no derivar a un territorio donde la multiplicidad sea utilizada como forma de aniquilar el futuro simbólico de lo femenino y donde podamos seguir "cuestionando» la diferencia sexual no como algo terminado y definitivo que "superar», sino como un proceso que nunca estuvo cerrado y sobre el que podemos intervenir» ${ }^{42}$.

Vemos, por tanto, que la clave radica en la continuidad del modelo sexista. Un modelo que se repite en la re-recreación de los submundos virtuales dentro del ciberespacio, como en Second life (donde es frecuente el sexo con mujeres, en un contexto a veces contradictorio con una autoridad nada diáfana en este asunto) o en los videojuegos, que se enmarcan en el imaginario masculino. Un mundo virtual donde los desprotegidos, mujeres y niños, son víctimas, por ejemplo, en lo que se refiere a la pornografía, que se muestra como un método de control sobre el cuerpo, a través de las imágenes que se difunden e intercambian en Internet.

En suma, el ciberespacio es un sitio de pugna, quizá como apunta Wyatt ${ }^{43}$, donde feminidad, masculinidad y tecnología no son categorías unitarias, sino que contienen múltiples posibilidades para ser construidas en relación con los demás.

41. Ibíd.

42. Ibíd.

43. WYATT, Sally: Op. cit. 


\section{CONCLUSIONES Y DISCUSIÓN}

La identidad es un tema candente en nuestros días, no sólo por fenómenos de la globalización, sino también por la presencia del ciberespacio. Junto a otros espacios, como el mediático, se presenta como una pieza clave para entendernos a nosotros mismos y entender a los demás. Posiblemente, se constituye también en una mirada desde donde comprender la realidad social, y que impone su propia lógica.

Existen algunos indicios, quizá en ocasiones contradictorios, que indican la presencia, en la sociedad actual, de múltiples identidades y cada vez más fragmentadas e inestables. No obstante, parece que algunas identidades muestran una clara resistencia ante este proceso, aunque sean de naturaleza diametralmente opuesta y por razones diferentes, como es el caso de la identidad de género y la identidad nacional. Desde la identidad de género, Internet se muestra como una tecnología que, al mismo tiempo, no escapa a la dominación del hombre, pero representa una oportunidad con una gran cantidad de variantes. En definitiva, un dominio de posibilidades para no sucumbir a la aplastante presencia de otras identidades.

Desde una perspectiva global, posiblemente, Internet y las tecnologías digitales propicien el grado adecuado de interferencia entre las identidades. Se debieran construir zonas donde sujetos y comunidades de procedencias culturales diferentes, pero con una determinada identidad tecnológica, puedan dialogar, lo que tendría consecuencias en diferentes contextos, también en el de la memoria.

Desde un punto de vista metodológico, quisiéramos finalizar destacando la idea de que los fenómenos aquí tratados deben ser estudiados, en un cruce interdisciplinar, a luz de diferentes ópticas como la de los estudios de género, la sociología, la economía política, los estudios culturales, y la comunicación, entre otras.

\section{BIBLIOGRAFÍA}

AGUILERA VALPUESTA, Francisco: «Sociabilidad virtual, vínculo social y construcción de la identidad. El Caso de la comunidad virtual El Rincón Chileno», II Congreso online del OCS, 2004. En www.cibersociedad.com/congres2004.

ANDREWS, Margaret: "Ethics, Gender and the Internet: An exploration of some Spanish feminists' praxis», Journal of Spanish Cultural Studies, 7:1 (2006), pp. 37-49.

BACH, Marta; Altés, Elvira; GALLEGo, Juana (eds.): El sexo de la noticia, Barcelona, Icaria, 2002.

BAILEY, Steven: Media audiences and identity. Self-construction in the fan experience, New York, Palgrave MacMillan, 2005.

BARKER, Chris: Televisión, globalización e identidades culturales, Barcelona, Paidós, 2003.

BIJKER, Wiebe E. y LAW, John (eds): Shaping Technology / building Society: Studies in Sociotechnical Change, Cambridge, MIT Press, 1992.

CampBell, Alex: "The Search for authenticity», New Media \& Society, 8:2 (2006), pp. 269-294.

Carter, Cynthia (ed): News, Gender and Power, Londres, Routledge, 1998.

CASTElls, Manuel: "Globalización e Identidad», Cuadernos del Mediterráneo, 5 (2005), pp. 11-20. En http://www.iade.org.ar/uploads/c87bbfe5-38f7-d1bb.pdf

- The Information Age, Oxford, Blackwell, 1996. 
CockBurn, Cynthia: Gender and Technology in the Making, Londres, Sage, 1993.

- Machinery of Dominance. Women, Men and Technical Know-How, Londres, Pluto Press, 1985.

CRUZ, Manuel: Las malas pasadas del pasado. Identidad, responsabilidad, historia, Barcelona, Anagrama, 2005.

FIRESTONE, Shulamith: The Dialectic of Sex, New York, Morrow, 1970.

GALlego, Juana: La prensa por dentro, Barcelona, Libros de la Frontera, 2002.

GARCÍA GUTIÉRREZ, Antonio: Fijaciones. Estudios críticos sobre politicas, culturas y tecnologías de la memoria, Madrid, Biblioteca Nueva, 2005.

HARAWAY, Donna: "Manifesto for cyborgs», The Socialist Review, 7 (1985), pp. 115-135.

JIMÉNEZ CORTÉS, Rocío: "Internet y educación: La gestión de la identidad cultural de género", $1^{\circ}$ Congreso Online del Observatorio para la Cibersociedad, (2002). En http:// www.cibersociedad.net/congreso/comms/g15jimenez.pdf

KENNEDY, Helen: "Beyond anonymity, or future directions for internet identity research", New media \& Society, 8:6 (2006), pp. 859-876.

LARRONDO URETA, Ainara: "La Red al servicio de las mujeres. Aproximación a la relación mujer y medios de comunicación en Internet», Estudios sobre el Mensaje Periodístico, 11 (2005), pp. 375-392.

LIE, Merete (ed): He, She and It Revisited. New Perspectives on Gender in the Information Society, Oslo, Gyldendal, 2003.

MORLEY, David y RoBIns, Kevin: Spaces of identity. Global media, Electronic Landscapes and Cultural Boundaries, London, Routledge, 2004.

Plant, Sadie: Zeros + Ones, Londres, Fourth Estate, 1997.

RORTY, Richard: Objetividad, relativismo y verdad: escritos filosóficos, Barcelona, Paidós, 1996.

SAMPEDRO, Víctor: «Identidades mediáticas. La lógica del régimen de visibilidad contemporánea", Sphera Pública, 4 (2004), pp. 17-36.

SANZ GONZÁLEZ, Verónica: «Las tecnologías de la información desde el punto de vista de género: posturas y propuestas desde el feminismo", Isegoría, 34 (2006), pp. 193-208.

SOLANAS, Valerie: The SCUM Manifesto, Londres, Verso, 2004.

TetzlafF, David: "Y-Ho-Ho and a Server of Warez: Internet Software Piracy and the new global information economy", en A. Herman and T. Swiss (eds.) The World Wide Web and Contemporary Cultural Theory, London, Routledge, 2000, pp. 99-126.

TURKLE, Sherry: La vida en la pantalla. La construcción de la identidad en la era de Internet, Barcelona, Paidós, 1997.

ValkenburG, Patti M.; Schouten, Alexander P. and Peter, Jochen: "Adolescents' identity experiments on the internet", New Media \& Society, 7:3 (2005), pp. 383-402.

VATTIMO, Gianni: La sociedad transparente, Barcelona, Paidós, 1990.

VERGARA, Enrique: «Medios de comunicación y globalización: destrucción o reconstrucción de identidades culturales», Ànalisi, 33 (2006), pp. 95-105.

VIRILO, Paul: El cibermundo. La política de lo peor, Madrid, Cátedra, 1999.

WajCMAn, Judy: Technofeminism, Cambridge, Polity Press, 2004.

WYATT, Sally: "Feminism, technology and the information society. Learning from the past, imagining the future», Information, Communication \&Society, 11:1 (2008), pp. 111-130.

ZAFRA, Remedios: "Apuntes sobre feminismo y subjetividad en Internet», Mujer y cultura visual. En http://2-red.net/mcv/pensamiento/tx/text_rz6.html

Zoonen, Van Liesbet: Feminist Media Studies, London, Sage, 1994.

Fecha de recepción: 01-04-2008

Fecha de aceptación: 11-04-2008 